Archive for

Organic Chemistry

Arkivoc 2019, part vi, 325-335

\title{
Pyridinium bromide as a mediator in electrochemical reactions: the preparation of cyclopropane-1,1-dicarbonitriles
}

\author{
Anatoly N. Vereshchagin,* Evgeniya O. Dorofeeva, Michail N. Elinson, and Mikhail P. Egorov
}

N. D. Zelinsky Institute of Organic Chemistry, Russian Academy of Sciences, 119991 Moscow, Russian

Federation

Email: vereshchagin@ioc.ac.ru

Received 08-07-2019

Accepted 11-24-2019

Published on line 12-25-2019

\section{Abstract}

Pyridinium bromide has been tested as a new mediator for electrochemical transformations in methanol and acetonitrile. An efficient protocol for the synthesis of cyclopropanes via the electrochemical transformations of alkylidenemalononitriles and $\mathrm{C}-\mathrm{H}$ acids (malononitrile, malonic ester, $\mathrm{N}, \mathrm{N}$-dimethylbarbituric acid, pyrazolin-5-ones) has been developed. The chemistry proceeds in a simple undivided cell under constant current conditions employing a substoichiometric amount of $\mathrm{PyHBr}$ that serves both as a redox catalyst and a supporting electrolyte; in this manner additional conducting salt is not required.

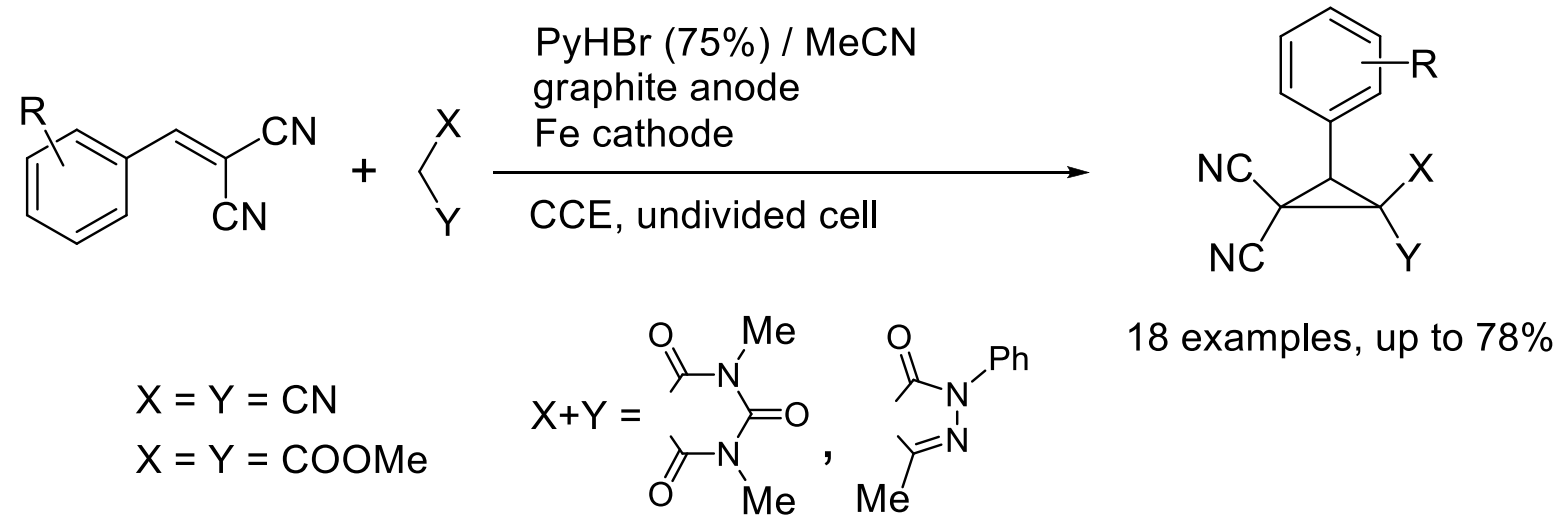

Keywords: Electrosynthesis, mediators, pyridinium bromide, $\mathrm{C}-\mathrm{H}$ acids, cyclopropanes 


\section{Introduction}

Electrosynthesis is a competitive method in modern organic chemistry. ${ }^{1-4}$ The importance of electrochemical synthesis lies in its great and, in some cases, unique possibilities for performing various transformations of organic compounds. ${ }^{5}$

In recent decades, indirect electrooxidation of organic compounds has been the subject of intensive studies. ${ }^{6,7}$ Halides are popular inorganic mediators. ${ }^{8-10}$ Their advantages include accessibility and low cost, as well as the fact that the majority of the processes catalyzed by these mediators are performed in an undivided cell.

$\mathrm{C}-\mathrm{H}$ acids are convenient synthons for the preparation of different classes of compounds by indirect electrooxidation in the presence of alkali halides as mediators. Electrochemical transformations involving different types of $\mathrm{C}-\mathrm{H}$ acids were studied. These processes are carried out in an undivided cell at constant current. Electrolysis of malononitrile or malonic esters in alcohols affords cyclopropanes. ${ }^{11,12}$ Multicomponent transformations of malonic acid derivatives using electrolysis media lead to the stereoselective formation of polysubstituted three-carbon rings. ${ }^{13-15}$ Cross-dehydrogenative coupling of $\beta$-diketones or $\beta$-ketoesters ( $\mathrm{C}-\mathrm{H}$ reagents) with carboxylic acids (O-H reagents) results in selective formation of intermolecular $\mathrm{C}-\mathrm{O}$ coupling products. ${ }^{16}$ Cyclic $\beta$-diketones enter into electrochemically induced multicomponent reactions with formation of pyrans ${ }^{17,18}$ and furans. ${ }^{19}$

Electrochemical processes involving heterocyclic $\mathrm{C}-\mathrm{H}$ acids are of considerable interest, since they allow the synthesis of a various types of heterocyclic compounds with a wide range of biological and pharmaceutical properties. $^{20}$ The $2,4,6(1 H, 3 H, 5 H)$-pyrimidinetriones, also called barbiturates (derivatives of barbituric acid), are a well-known class of drugs that act as central nervous system depressants, and by virtue of this, they produce a wide spectrum of effects, ranging from mild sedation to anaesthesia. ${ }^{21,22}$ An electrochemical strategy for the synthesis of pyrano[2,3-d]pyrimidine-2,4,7-triones is described, using an electrogenerated anion of barbituric acid in a one-pot, three component condensation of an aromatic aldehyde, Meldrum's acid (one more heterocyclic $\mathrm{C}-\mathrm{H}$ acid) and barbituric acid in ethanol in an undivided cell and in the presence of sodium bromide. ${ }^{23}$

Among 2,4,6(1H,3H,5H)-pyrimidinetriones, spirobarbiturates are a particular class of compounds with pronounced pharmacological and physiological activity. ${ }^{24-26}$ Our previous work described the indirect electrocatalytic transformation involving barbituric acids with formation of spiro compounds: furo[2,3- $d$ ]pyrimidines, ${ }^{27}$ terpyrimidines ${ }^{28}$ and spirobicycles containing cyclopropane and pyrimidine fragments. ${ }^{29} \mathrm{~A}$ spirocyclopropyl moiety joined to a heterocyclic ring has attracted particular attention due to a wide number of pronounced pharmacological applications. ${ }^{30,31}$

Pyrazolones are another important class of heterocycles possessing important biological properties. ${ }^{32}$ The $^{2}$ $\mathrm{C}-\mathrm{H}$ acid 3-methyl-1-phenyl-2-pyrazolin-5-one (edaravone) is a neuroprotective agent. ${ }^{33}$ The indirect electrooxidation of edavarone and analogues in the presence of sodium halides as mediators in alcohols has been described. Thus, Zeng et al. have carried out dehydrodimerization and dehydrotrimerization of pyrazol-5ones. ${ }^{34}$ Electrolysis of edavarone with aromatic aldehydes leads to the formation of 4,10-dimethyl-2,8,11triphenyl-2,3,8,9-tetraazadispiro[4.0.4.1] undeca-3,9-diene-1,7-diones, ${ }^{35}$ which was recently patented as an advanced glycation end product (AGE) formation inhibitor intended for treatment of human schizophrenia. ${ }^{36}$

Despite the advantages and significant achievements described, the use of alkali metal halides as a mediator in alcohol solutions for carrying out electrochemical transformations of organic compounds has several disadvantages. Thus, the need to use methanol or ethanol as a solvent does not allow the transformation of organic compounds which are insoluble or poorly soluble in alcohol, which limits the choice 
of the starting compounds and narrows the scope of the application of the electrocatalytic methodology. Further, there are also significant limitations due to the occurrence of a number of side reactions. Thus, the co-electrolysis of cyclohexylidenemalononitrile and malononitrile in ethanol in the presence of sodium bromide results in formation of the expected tetracyanocyclopropane with a tricyclic pyrroline as a side product. ${ }^{37}$ This result is a consequence of the ethoxide attack on the cyano-groups of the cyclopropane. To avoid this side reaction, it is necessary to use an aprotic solvent such as acetonitrile, dimethylformamide or dimethylsulfoxide. However, sodium halides do not dissolve in these solvents.

As is well known, quaternary ammonium salts are widely used in electrochemistry as supporting electrolytes. Recently Little, Zeng et al. reported the development of an electrochemically initiated oxidative amination of benzoxazoles ${ }^{38}$ and sulfonamides $^{39}$ using tetraalkylammonium halides and ammonium iodide as redox catalysts, respectively.

Now we present pyridinium bromide as a new mediator in electrochemical transformations involving benzylidenemalononitriles and $\mathrm{C}-\mathrm{H}$ acids.

\section{Results and Discussion}

We started using benzylidenemalononitrile $\mathbf{1 a}$ and malononitrile $\mathbf{2 a}$ as model substrates and performed a constant current electrolysis at $100 \mathrm{~mA} / \mathrm{cm}^{2}$ in an undivided cell, using a graphite rod (area $5 \mathrm{~cm}^{2}$ ) as the anode and a Fe plate (area $5 \mathrm{~cm}^{2}$ ) as the cathode (Scheme 1, Table 1).

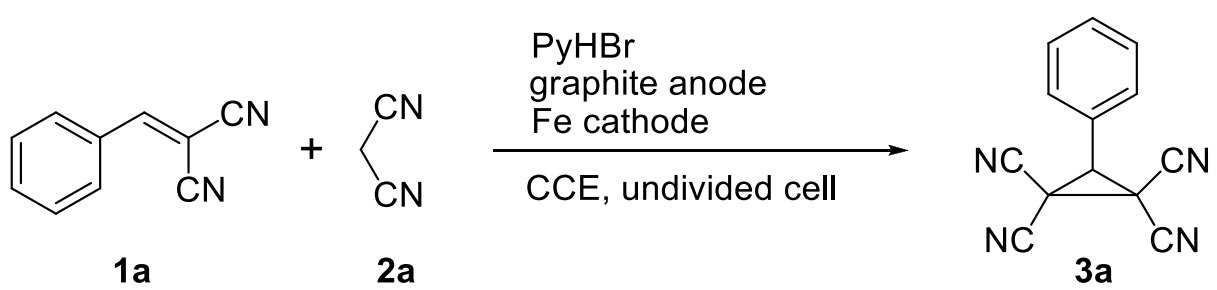

\section{Scheme 1}

Initially, we optimized the catalytic efficacy of $\mathrm{PyHBr}$ in methanol (entries 1-10). The current density of 50 $\mathrm{mA} \mathrm{cm}{ }^{-2}, 0.75$ equivalent of $\mathrm{PyHBr}, 20^{\circ} \mathrm{C}, 2.2 \mathrm{~F} \mathrm{~mol}^{-1}$ were found to be the optimum conditions (entry 5). Using smaller amounts of $\mathrm{PyHBr}$ in the electrolysis leads to the formation of $\mathbf{3 a}$ in lower yields (entries 1,2 ). Apparently, this is due to the incomplete regeneration of the mediator under electrolysis conditions. Raising the current density to $100 \mathrm{~mA} \mathrm{~cm}^{-2}$ (entry 4) or the temperature to $30{ }^{\circ} \mathrm{C}$ (entry 8 ) also result in a decrease of the yield, possibly due to the acceleration of undesired direct electrochemical processes leading to the oligomerization of the reactants. Low yields and incomplete conversion of starting compounds were observed when electrolysis was carried out at $10^{\circ} \mathrm{C}$ (entry 7). Ethanol is a slightly less effective solvent than methanol, in view of the yield. $\mathrm{PyHBr}$ is soluble in aprotic solvents. Acetonitrile, DMF and DMSO were tested as solvents for electrolysis (Table 1, entries 12-14). It was found that acetonitrile is also effective in the synthesis of $3 a$ under electrolysis conditions (entry 12 ). 
Table 1. Initial optimization of the electrochemical synthesis of tetracyanocyclopropane $\mathbf{3 a}$ using $\mathrm{PyHBr}$ as mediator $^{a}$

\begin{tabular}{ccccccc}
\hline Entry & Solvent & $\begin{array}{c}\text { Equiv. of } \\
\text { PyHBr }\end{array}$ & $\mathrm{T} /{ }^{\circ} \mathrm{C}$ & $\begin{array}{c}\text { Current } \\
\text { density/ } \\
\mathrm{mA} \mathrm{cm}^{-2}\end{array}$ & $\begin{array}{c}\text { Electricity } \\
\text { passed/ } \\
\mathrm{F} \mathrm{mol}^{-1}\end{array}$ & $\begin{array}{c}\text { Yield of 3a } \\
(\%)^{\mathrm{b}}\end{array}$ \\
\hline 1 & $\mathrm{MeOH}$ & 0.3 & 20 & 100 & 2.0 & $25^{\mathrm{c}}$ \\
2 & $\mathrm{MeOH}$ & 0.5 & 20 & 100 & 2.0 & 42 \\
3 & $\mathrm{MeOH}$ & 0.75 & 20 & 100 & 2.0 & 60 \\
4 & $\mathrm{MeOH}$ & 1.0 & 20 & 100 & 2.0 & 60 \\
5 & $\mathrm{MeOH}$ & 0.75 & 20 & 100 & 2.2 & 68 \\
6 & $\mathrm{MeOH}$ & 0.75 & 20 & 100 & 2.5 & 61 \\
7 & $\mathrm{MeOH}$ & 0.75 & 10 & 100 & 2.2 & 44 \\
8 & $\mathrm{MeOH}$ & 0.75 & 30 & 100 & 2.2 & $32^{\mathrm{c}}$ \\
9 & $\mathrm{MeOH}$ & 0.75 & 20 & 50 & 2.2 & 72 \\
10 & $\mathrm{MeOH}$ & 0.75 & 20 & 25 & 2.2 & 68 \\
11 & $\mathrm{EtOH}$ & 0.75 & 20 & 50 & 2.2 & 62 \\
12 & $\mathrm{MeCN}$ & 0.75 & 20 & 50 & 2.2 & 72 \\
13 & $\mathrm{DMF}$ & 0.75 & 20 & 50 & 2.2 & $35^{\mathrm{c}}$ \\
14 & $\mathrm{DMSO}$ & 0.75 & 20 & 50 & 2.2 & $25^{\mathrm{c}}$ \\
\hline
\end{tabular}

a Benzylidenemalononitrile $1 \mathrm{a}(10 \mathrm{mmol})$, malononitrile $2 \mathrm{a}(10 \mathrm{mmol}), 20 \mathrm{~mL}$ of solvent, undivided cell.

b Isolated yield. ' NMR data.

From the results described above, we conclude that the optimal reaction conditions require using $75 \mathrm{~mol} \%$ of $\mathrm{PyHBr}$ as mediator and supporting electrolyte, and $50 \mathrm{~mA} \mathrm{~cm}{ }^{-2}$ of current density at $20{ }^{\circ} \mathrm{C}$. The reaction is best performed in an undivided cell using methanol or acetonitrile as the solvent without the need for additional conducting salt. It should be noted that $\mathrm{PyHBr}$ is an effective mediator both in methanol and in acetonitrile with a current density of $50 \mathrm{~mA} \mathrm{~cm}$. . Previous electrochemical methods for the synthesis of tetracyanocyclopropanes $\mathbf{3}$ were carried out utilizing alkali metal halides as mediators with a current density of $100 \mathrm{~mA} \mathrm{~cm}{ }^{-2}{ }^{11}$ However, the existing method is only applicable in alcohols, since alkali halides are insoluble in aprotic solvents such as acetonitrile. As a result, we have now the opportunity to conduct an electrochemical reaction in a new medium.
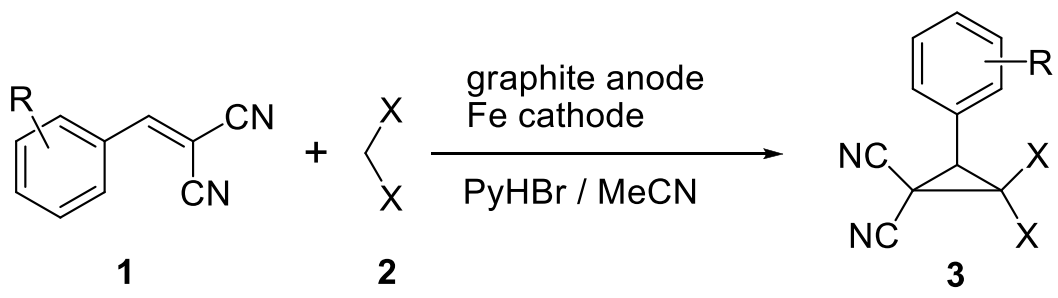

\section{Scheme 2}

Under the optimal conditions, as determined above, we then studied the scope and generality of the protocol by examining reactions of benzylidenemalononitriles 1 (with both electron-withdrawing and electrondonating substituents on the aromatic ring), and with different types of $\mathrm{C}-\mathrm{H}$ acids: acyclic malononitrile $2 \mathrm{a}$ or 
dimethyl malonate $\mathbf{2 b}$ (Scheme 2, Table 2); heterocyclic 1,3-dimethylbarbituric acid 2c and edavarone (3methyl-1-phenyl-2-pyrazolin-5-one) 2d (Scheme 3, Table 3).

Table 2. Electrochemical synthesis of cyclopropanes $\mathbf{3}$ using noncyclic $\mathrm{C}-\mathrm{H}$ acids $\mathbf{2 a , b}$ and $\mathrm{PyHBr}$ as mediator $^{a}$

\begin{tabular}{|c|c|c|c|c|c|c|}
\hline Entry & Olefin & $\mathrm{R}$ & $\mathrm{C}-\mathrm{H}$ acid & $x$ & Cyclopropane & Yield of $3,(\%)^{b}$ \\
\hline 1 & $1 a$ & $\mathrm{H}$ & $2 a$ & $\mathrm{CN}$ & $3 a$ & 72 \\
\hline 2 & $1 b$ & 4-Me & $2 a$ & $\mathrm{CN}$ & $3 b$ & 66 \\
\hline 3 & $1 c$ & $3-\mathrm{Br}$ & $2 a$ & $\mathrm{CN}$ & $3 c$ & 58 \\
\hline 4 & $1 d$ & $4-\mathrm{NO}_{2}$ & $2 a$ & $\mathrm{CN}$ & $3 d$ & 48 \\
\hline 5 & $1 a$ & $\mathrm{H}$ & $2 b$ & coOMe & $3 e$ & 67 \\
\hline 6 & $1 b$ & 4-Me & $2 b$ & coOMe & $3 f$ & 63 \\
\hline 7 & $1 c$ & $3-\mathrm{Br}$ & $2 b$ & COOMe & $3 g$ & 64 \\
\hline 8 & $1 d$ & $4-\mathrm{NO}_{2}$ & $2 b$ & coOMe & $3 h$ & 38 \\
\hline
\end{tabular}

a Benzylidenemalononitrile $1(10 \mathrm{mmol}), \mathrm{C}-\mathrm{H}$ acid $2(10 \mathrm{mmol}), 20 \mathrm{~mL}$ of acetonitrile, undivided cell, $20^{\circ} \mathrm{C}$, $2.2 \mathrm{~F} \mathrm{~mol}^{-1}$, current density $50 \mathrm{~mA} \mathrm{~cm}^{-2}$. ${ }^{\text {b }}$ Isolated yield.

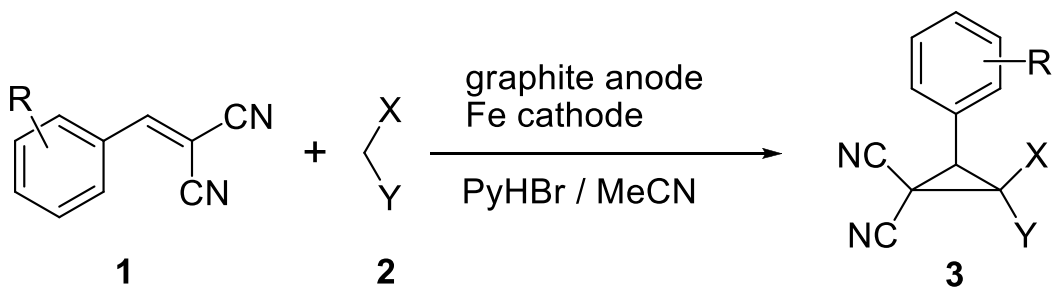

\section{Scheme 3}

Table 3. Electrochemical synthesis of cyclopropanes $\mathbf{3}$ using heterocyclic $\mathrm{C}-\mathrm{H}$ acids $\mathbf{2 c}, \mathbf{d}$ and $\mathrm{PyHBr}$ as mediator $^{a}$

\begin{tabular}{|c|c|c|c|c|c|c|}
\hline Entry & Olefin & $\mathrm{R}$ & $\mathrm{C}-\mathrm{H}$ acid & $X+Y$ & Cyclopropane & Yield of $3,(\%)^{b}$ \\
\hline 1 & $1 a$ & $\mathrm{H}$ & $2 c$ & & $3 \mathbf{i}$ & 78 \\
\hline 2 & $1 b$ & 4-Me & $2 c$ & & $3 \mathbf{j}$ & 65 \\
\hline 3 & $1 c$ & $3-B r$ & $2 c$ & & $3 k$ & 74 \\
\hline 4 & $1 d$ & $4-\mathrm{NO}_{2}$ & $2 c$ & & 31 & 54 \\
\hline 5 & $1 e$ & 3-OMe & 2c & & $3 m$ & 66 \\
\hline 6 & $1 a$ & $\mathrm{H}$ & $2 d$ & & $3 n$ & $55(4: 1)^{c}$ \\
\hline 7 & $1 b$ & 4-Me & $2 d$ & & 30 & $62(5: 2)^{c}$ \\
\hline 8 & $1 c$ & $3-\mathrm{Br}$ & $2 d$ & & $3 p$ & $59(3: 1)^{c}$ \\
\hline 9 & $1 d$ & $4-\mathrm{NO}_{2}$ & $2 d$ & & $3 q$ & $35(3: 2)^{c}$ \\
\hline 10 & $1 e$ & 3-OMe & $2 d$ & & $3 r$ & $64(6: 1)^{c}$ \\
\hline
\end{tabular}

a Benzylidenemalononitrile 1 (10 mmol), C-H acid $2(10 \mathrm{mmol}), 20 \mathrm{~mL}$ of acetonitrile, undivided cell, $20^{\circ} \mathrm{C}$, $2.2 \mathrm{~F} \mathrm{~mol}^{-1}$, current density $50 \mathrm{~mA} \mathrm{~cm}^{-2} .{ }^{\mathrm{b}}$ Isolated yield. ${ }^{\mathrm{c}}$ Ratio of izomers. 
4-Methyl-7-oxo-2,6-diaryl-5,6-diazaspiro[2.4]hept-4-ene-1,1-dicarbonitriles $3 \mathrm{~m}$-3p were obtained as mixtures of diastereomers. The structures of the major $(2 R S, 3 S R) \mathbf{3 m - 3 p}$ isomers were established earlier on the data of NOESY. ${ }^{40}$ It should be noted that sodium bromide in methanol is a more effective mediator than $\mathrm{PyHBr}$ in acetonitrile for the electrochemical synthesis of spirocyclopropanes 3i-3p. ${ }^{29,40}$ Apparently this is due to the particularity of the reaction mechanism (Scheme 4).

A possible mechanism for the current transformation is proposed (Scheme 1). Bromine is formed at the anode and can be observed by its color. Deprotonation of the pyridinium cation at the cathode leads to the formation of pyridine. The evolution of hydrogen at the cathode is observed, especially when electrolysis is conducted without stirring of the reaction mixture. Deprotonation of the $\mathrm{C}-\mathrm{H}$ acid by pyridine in solution and further bromination of the $\mathrm{C}-\mathrm{H}$ acid anion forms the $\mathrm{C}-\mathrm{H}$ acid $\mathbf{A}$. Next, the ylide $\mathbf{B}$ is formed from the intermediate $\mathbf{A}$ by action of pyridine. Finally, addition of $\mathbf{B}$ to alkylidenemalononitrile gives rise to cyclopropane 3. The last step was postulated by Abaszadeh in the chemical synthesis of cyclopropanes from pyridinium ylides and benzylidenemalononitriles. ${ }^{41}$

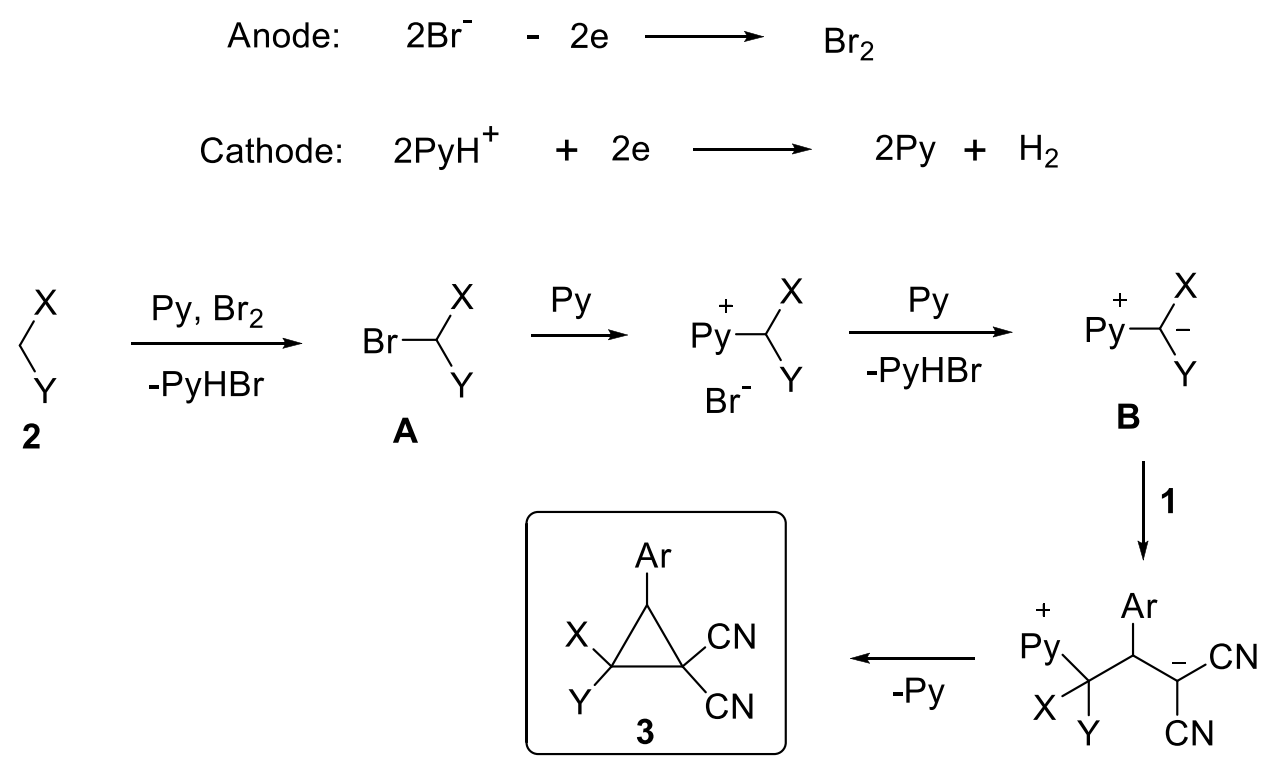

\section{Scheme 4}

\section{Conclusions}

Summarizing, we have tested a novel mediator for electrochemical transformations. The electrochemical synthesis was performed under high constant current density in a simple undivided cell using a substoichiometric amount of pyridinium bromide as mediator. Utilization of pyridinium bromide allows to perform the electrolysis both in alcohols and aprotic solvents such as acetonitrile. After optimization of the reaction conditions, the tetracyanocyclopropanes, dimethyl 2,2-dicyano-3-arylcyclopropane-1,1-dicarboxylates and two types of spirocyclopropanes: 5,7-dimethyl-4,6,8-trioxo-2-aryl-5,7-diazaspiro[2.5]octane-1,1dicarbonitriles and 4-methyl-7-oxo-2,6-diaryl-5,6-diazaspiro[2.4]hept-4-ene-1,1-dicarbonitriles have been synthesized. The reactions proceed in a simple undivided cell employing a substoichiometric amount of $\mathrm{PyHBr}$ that serves both as a mediator and a supporting electrolyte; in this manner additional conducting salt is not required. The developed technique allows the synthesis of gram-scale amounts of the target compounds. 


\section{Experimental Section}

General. All melting points were measured with a Gallenkamp melting point apparatus. ${ }^{1} \mathrm{H}$ NMR and ${ }^{13} \mathrm{C}$ NMR were recorded with a Bruker AM300 at ambient temperature in DMSO-d $d_{6}$ or $\mathrm{CDCl}_{3}$ solutions with working frequencies of $300.13 \mathrm{MHz}$ and $75.47 \mathrm{MHz}$ respectively. Chemical shifts values are given in $\delta$ scale relative to $\mathrm{Me}_{4} \mathrm{Si}$. IR spectra were recorded with a Bruker ALPHA-T FT-IR spectrometer in $\mathrm{KBr}$ pellets. Mass-spectra $(E I=70 \mathrm{eV})$ were recorded with a Finningan MAT INCOS 50 spectrometer. High-resolution mass spectrometry (HRMS) (electrospray ionization, ESI) was measured on a BrukermicroTOF II instrument; external or internal calibration was done with an Electrospray Calibrant Solution (Fluka). Benzylidenemalononitriles 1 were synthesized by the condensation of the corresponding aromatic aldehydes and malononitrile via the Knoevenagel condensation. ${ }^{15}$

General procedure. An undivided cell was equipped with a graphite anode (area $5 \mathrm{~cm}^{2}$ ) and Fe cathode (area 5 $\mathrm{cm}^{2}$ ) and connected to a DC regulated power supply. To the cell were added the desired benzylidenemalononitrile 1 (10 mmol), C-H acid $2(10 \mathrm{mmol}), \mathrm{PyHBr}(7.5 \mathrm{mmol})$, and $20 \mathrm{~mL}$ of acetonitrile. The mixture was electrolyzed $\left(2.2 \mathrm{~F} \mathrm{~mol}^{-1}\right)$ using constant current conditions $\left(50 \mathrm{~mA} / \mathrm{cm}^{2}\right)$ at $20{ }^{\circ} \mathrm{C}$ under magnetic stirring. When the reaction was completed, the solvent was removed under reduced pressure. The residue was poured into water, and the product was then extracted with dichloromethane $(3 \times 20 \mathrm{~mL})$, dried over $\mathrm{Na}_{2} \mathrm{SO}_{4}$, and concentrated in vacuum. The residue was purified by crystallization from methanol to afford the desired cyclopropane 3.

3-Phenyl-1,1,2,2-tetracyanocyclopropane (3a). White solid; $1.57 \mathrm{~g}$ (72\%); mp 226-228 ${ }^{\circ} \mathrm{C}$ (lit. ${ }^{42} \mathrm{mp} 229-230$ $\left.{ }^{\circ} \mathrm{C}\right) .{ }^{1} \mathrm{H}$ NMR (DMSO- $\left.d_{6}\right): \delta 5.10(1 \mathrm{H}, \mathrm{s}, \mathrm{CH}), 7.48-7.80(5 \mathrm{H}, \mathrm{m}, \mathrm{Ar}) \mathrm{ppm}$.

3-(4-Methylphenyl)-1,1,2,2-tetracyanocyclopropane (3b). White solid; $1.53 \mathrm{~g}(66 \%) ; \mathrm{mp} 224-227{ }^{\circ} \mathrm{C}$ (lit. ${ }^{42}$ mp 226-229 $\left.{ }^{\circ} \mathrm{C}\right) .{ }^{1} \mathrm{H}$ NMR (DMSO-d 6 ): $\delta 2.31\left(3 \mathrm{H}, \mathrm{s}, \mathrm{CH}_{3}\right), 5.20(1 \mathrm{H}, \mathrm{s}, \mathrm{CH}), 7.28(2 \mathrm{H}, \mathrm{d}, J 8.0 \mathrm{~Hz}, \mathrm{Ar}), 7.65(2 \mathrm{H}, \mathrm{d}, J$ $8.0 \mathrm{~Hz}, \mathrm{Ar}) \mathrm{ppm}$.

3-(3-Bromophenyl)-1,1,2,2-tetracyanocyclopropane (3c). White solid; $1.72 \mathrm{~g}$ (58\%); mp $184-186{ }^{\circ} \mathrm{C}$ (lit. ${ }^{42}$ $\left.\mathrm{mp} 186-187^{\circ} \mathrm{C}\right) .{ }^{1} \mathrm{H}$ NMR (DMSO- $\left.d_{6}\right): \delta 5.31(1 \mathrm{H}, \mathrm{s}, \mathrm{CH}), 7.44(1 \mathrm{H}, \mathrm{t}, J 8.5 \mathrm{~Hz}, \mathrm{Ar}), 7.68(1 \mathrm{H}, \mathrm{d}, J 8.5 \mathrm{~Hz}, \mathrm{Ar})$, 7.87(1H, d, J $8.5 \mathrm{~Hz}, \mathrm{Ar}), 8.22(1 \mathrm{H}, \mathrm{s}, \mathrm{Ar}) \mathrm{ppm}$.

3-(4-Nitrophenyl)-1,1,2,2-tetracyanocyclopropane (3d). White solid; $1.26 \mathrm{~g} \mathrm{(48 \% );} \mathrm{mp} 232-234{ }^{\circ} \mathrm{C}$ (lit. ${ }^{42}$ $\left.\mathrm{mp} 232-234^{\circ} \mathrm{C}\right) .{ }^{1} \mathrm{H}$ NMR (DMSO- $\left.d_{6}\right): \delta 5.52(1 \mathrm{H}, \mathrm{s}, \mathrm{CH}), 8.20(2 \mathrm{H}, \mathrm{d}, J 8.8 \mathrm{~Hz}, \mathrm{Ar}), 8.35(2 \mathrm{H}, \mathrm{d}, J 8.8 \mathrm{~Hz}, \mathrm{Ar}) \mathrm{ppm}$.

Dimethyl 2,2-dicyano-3-phenylcyclopropane-1,1-dicarboxylate (3e). White solid; $1.90 \mathrm{~g}$ (67\%); mp 124-126 ${ }^{\circ} \mathrm{C}$. (lit. $\left.{ }^{12} \mathrm{mp} 126-128{ }^{\circ} \mathrm{C}\right) .{ }^{1} \mathrm{H}$ NMR (DMSO-d $): \delta 3.75\left(\mathrm{~s}, 3 \mathrm{H}, \mathrm{OCH}_{3}\right), 3.92\left(\mathrm{~s}, 3 \mathrm{H}, \mathrm{OCH}_{3}\right), 4.41(\mathrm{~s}, 1 \mathrm{H}, \mathrm{CH}), 7.35-$ $7.50(\mathrm{~m}, 5 \mathrm{H}, \mathrm{Ph}) \mathrm{ppm}$.

Dimethyl 2,2-dicyano-3-(4-methylphenyl)cyclopropane-1,1-dicarboxylate (3f). White solid; $1.87 \mathrm{~g}(63 \%) ; \mathrm{mp}$ 134-13135 ${ }^{\circ} \mathrm{C}$. (lit. $\left.{ }^{12} \mathrm{mp} 137-139{ }^{\circ} \mathrm{C}\right) .{ }^{1} \mathrm{H}$ NMR $\left(\mathrm{CDCl}_{3}\right): \delta 2.39\left(\mathrm{~s}, 3 \mathrm{H}, \mathrm{CH}_{3}\right), 3.79\left(\mathrm{~s}, 3 \mathrm{H}, \mathrm{OCH}_{3}\right), 3.96(\mathrm{~s}, 1 \mathrm{H}, \mathrm{CH})$, $3.98\left(\mathrm{~s}, 3 \mathrm{H}, \mathrm{OCH}_{3}\right), 7.18-7.30(\mathrm{~m}, 4 \mathrm{H}, \mathrm{Ar}) \mathrm{ppm}$.

Dimethyl 2,2-dicyano-3-(3-bromophenyl)cyclopropane-1,1-dicarboxylate (3g). White solid; $2.32 \mathrm{~g}(64 \%) ; \mathrm{mp}$ $110-111^{\circ} \mathrm{C}$ (lit $\left.{ }^{12} \mathrm{mp} 110-111^{\circ} \mathrm{C}\right) .{ }^{1} \mathrm{H}$ NMR $\left(\mathrm{CDCl}_{3}\right): \delta 3.80\left(\mathrm{~s}, 3 \mathrm{H}, \mathrm{OCH}_{3}\right), 3.95(\mathrm{~s}, 1 \mathrm{H}, \mathrm{CH}), 3.97\left(\mathrm{~s}, 3 \mathrm{H}, \mathrm{OCH}_{3}\right), 7.26-$ 7.35 (m, 2H, Ar), 7.50-7.55 (m, 2H, Ar) ppm.

Dimethyl 2,2-dicyano-3-(4-nitrophenyl)cyclopropane-1,1-dicarboxylate (3h). White solid; $1.25 \mathrm{~g}(38 \%) ; \mathrm{mp}$ 141-143 ${ }^{\circ} \mathrm{C}$. (lit. $\left.{ }^{12} \mathrm{mp} \mathrm{140-142}{ }^{\circ} \mathrm{C}\right) .{ }^{1} \mathrm{H}$ NMR $\left(\mathrm{CDCl}_{3}\right): \delta 3.85$ (s, 3H, OCH $), 4.02\left(\mathrm{~s}, 3 \mathrm{H}, \mathrm{OCH}_{3}\right), 4.05(\mathrm{~s}, 1 \mathrm{H}, \mathrm{CH})$, $7.62(2 \mathrm{H}, \mathrm{d}, J 8.0 \mathrm{~Hz}, \mathrm{Ar}), 8.30(2 \mathrm{H}, \mathrm{d}, J 8.0 \mathrm{~Hz}, \mathrm{Ar}) \mathrm{ppm}$. 
5,7-Dimethyl-4,6,8-trioxo-2-phenyl-5,7-diazaspiro[2.5]octane-1,1-dicarbonitrile (3i). White solid; $2.40 \mathrm{~g}$ (78\%); mp. $258-260{ }^{\circ} \mathrm{C}$ (lit. ${ }^{29} \mathrm{mp} 259-260{ }^{\circ} \mathrm{C}$ ). ${ }^{1} \mathrm{H}$ NMR (DMSO-d $): \delta 3.12\left(\mathrm{~s}, 3 \mathrm{H}, \mathrm{CH}_{3}\right.$ ), $3.27\left(\mathrm{~s}, 3 \mathrm{H}, \mathrm{CH}_{3}\right), 4.36(\mathrm{~s}$, $1 \mathrm{H}, \mathrm{CH}), 7.32-7.40(\mathrm{~m}, 3 \mathrm{H}, \mathrm{Ar}), 7.44-7.50(\mathrm{~m}, 2 \mathrm{H}, \mathrm{Ar}) \mathrm{ppm}$.

5,7-Dimethyl-2-(4-methylphenyl)-4,6,8-trioxo-5,7-diazaspiro[2.5]octane-1,1-dicarbonitrile (3j). White solid; $2.09 \mathrm{~g}(65 \%) ; \mathrm{mp} .203-204{ }^{\circ} \mathrm{C}$ (lit. ${ }^{29} \mathrm{mp} 203-204{ }^{\circ} \mathrm{C}$ ). ${ }^{1} \mathrm{H}$ NMR (DMSO-d $)$ ): $\delta 2.30\left(\mathrm{~s}, 3 \mathrm{H}, \mathrm{CH}_{3}\right), 3.12\left(\mathrm{~s}, 3 \mathrm{H}, \mathrm{CH}_{3}\right)$, $3.26\left(\mathrm{~s}, 3 \mathrm{H}, \mathrm{CH}_{3}\right), 4.29(\mathrm{~s}, 1 \mathrm{H}, \mathrm{CH}), 7.17$ (d, J $7.9 \mathrm{~Hz}, 2 \mathrm{H}, \mathrm{Ar}$ ), 7.33 (d, J 7.9 Hz, 2H, Ar) ppm.

2-(3-Bromophenyl)-5,7-dimethyl-4,6,8-trioxo-5,7-diazaspiro[2.5]octane-1,1-dicarbonitrile (3k). White solid; $2.86 \mathrm{~g}(74 \%) ; \mathrm{mp} .222-224{ }^{\circ} \mathrm{C}$ (lit. ${ }^{29} 220-222{ }^{\circ} \mathrm{C}$ ). ${ }^{1} \mathrm{H}$ NMR (DMSO-d $): \delta 3.12\left(\mathrm{~s}, 3 \mathrm{H}, \mathrm{CH}_{3}\right), 3.28\left(\mathrm{~s}, 3 \mathrm{H}, \mathrm{CH}_{3}\right), 4.39$ $(\mathrm{s}, 1 \mathrm{H}, \mathrm{CH}), 7.35(\mathrm{t}, J \mathrm{~J} .9 \mathrm{~Hz}, 1 \mathrm{H}, \mathrm{Ph}), 7.50-7.57(\mathrm{~m}, 2 \mathrm{H}, \mathrm{Ph}), 7.77-7.79(\mathrm{~m}, 1 \mathrm{H}, \mathrm{Ph}) \mathrm{ppm}$.

5,7-Dimethyl-2-(4-nitrophenyl)-4,6,8-trioxo-5,7-diazaspiro[2.5]octane-1,1-dicarbonitrile (3l). Yellowish solid; $1.90 \mathrm{~g}(54 \%) ; \mathrm{mp} .219-221{ }^{\circ} \mathrm{C}$ (lit. ${ }^{29} 219-221^{\circ} \mathrm{C}$ ). ${ }^{1} \mathrm{H}$ NMR (DMSO-d $)$ ): $\delta 3.12\left(\mathrm{~s}, 3 \mathrm{H}, \mathrm{CH}_{3}\right), 3.30\left(\mathrm{~s}, 3 \mathrm{H}, \mathrm{CH}_{3}\right), 4.55$ $(\mathrm{s}, 1 \mathrm{H}, \mathrm{CH}), 7.82(\mathrm{~d}, J 8.4 \mathrm{~Hz}, 2 \mathrm{H}, \mathrm{Ar}), 8.24(\mathrm{~d}, J 8.4 \mathrm{~Hz}, 2 \mathrm{H}, \mathrm{Ar})$.

5,7-Dimethyl-2-(3-methoxyphenyl)-4,6,8-trioxo-5,7-diazaspiro[2.5]octane-1,1-dicarbonitrile (3m). White solid; $2.23 \mathrm{~g}(66 \%) ; \mathrm{mp} .232-234{ }^{\circ} \mathrm{C} .{ }^{1} \mathrm{H}$ NMR (DMSO-d $)$ : $\delta 3.12\left(\mathrm{~s}, 3 \mathrm{H}, \mathrm{CH}_{3}\right), 3.26\left(\mathrm{~s}, 3 \mathrm{H}, \mathrm{CH}_{3}\right), 4.08(\mathrm{~s}, 1 \mathrm{H}, \mathrm{CH})$, $3.76\left(\mathrm{~s}, 3 \mathrm{H}, \mathrm{OCH}_{3}\right), 6.90-7.12$ (m, 2H, Ar), 7.20-7.44 (m, 2H, Ar) ppm; ${ }^{13} \mathrm{C} \mathrm{NMR}$ (DMSO-d $)$ ): $\delta$ 23.4, 28.4, 29.1, 40.1, $41.2,55.5,109.4,110.9,112.4,115.9,120.7,128.2,130.6,150.3,157.0,160.6,163.0$ ppm. IR (KBr): 768, 1396, 1424, 1440, 1684, 1708, $2248 \mathrm{~cm}^{-1}$. HRMS (ESI) $339.1093[\mathrm{M}+\mathrm{H}]^{+}$, calcd for $\mathrm{C}_{17} \mathrm{H}_{15} \mathrm{~N}_{4} \mathrm{O}_{4}: 339.1099$.

4-Methyl-7-oxo-2,6-diphenyl-5,6-diazaspiro[2.4] hept-4-ene-1,1-dicarbonitrile (3n). White solid; $1.79 \mathrm{~g}$ (55\%); diastereomeric ratio 4:1; mp 187-189 ${ }^{\circ} \mathrm{C}$ (lit. ${ }^{43} \mathrm{mp}$ 187-189 ${ }^{\circ} \mathrm{C}$ ). ${ }^{1} \mathrm{H}$ NMR (DMSO- $d_{6}$ ) major diastereoisomer: $\delta$ $1.71\left(\mathrm{~s}, 3 \mathrm{H}, \mathrm{CH}_{3}\right), 4.20(\mathrm{~s}, 1 \mathrm{H}, \mathrm{CH}), 7.38-7.52(\mathrm{~m}, 8 \mathrm{H}, \mathrm{Ar}), 7.95$ (d, J $\left.7.9 \mathrm{~Hz}, 2 \mathrm{H}, \mathrm{Ar}\right) \mathrm{ppm}$; minor diastereoisomer: $\delta 2.35\left(\mathrm{~s}, 3 \mathrm{H}, \mathrm{CH}_{3}\right), 4.94(\mathrm{~s}, 1 \mathrm{H}, \mathrm{CH}), 7.19-7.35(\mathrm{~m}, 8 \mathrm{H}, \mathrm{Ar}), 7.85(\mathrm{~d}, J 7.9 \mathrm{~Hz}, 2 \mathrm{H}, \mathrm{Ar}) \mathrm{ppm}$.

4-Methyl-2-(4-methylphenyl)-7-oxo-6-phenyl-5,6-diazaspiro[2.4]hept-4-ene-1,1-dicarbonitrile (3o). White solid; $2.10 \mathrm{~g}$ (62\%); diastereomeric ratio 5:2; $\mathrm{mp} 168-170{ }^{\circ} \mathrm{C}$ (lit. ${ }^{43} \mathrm{mp} 175-178{ }^{\circ} \mathrm{C}$ ). ${ }^{1} \mathrm{H}$ NMR (DMSO- $\left.d_{6}\right)$ major diastereoisomer: $\delta 1.88\left(\mathrm{~s}, 3 \mathrm{H}, \mathrm{CH}_{3}\right), 2.41\left(\mathrm{~s}, 3 \mathrm{H}, \mathrm{CH}_{3}\right), 3.93(\mathrm{~s}, 1 \mathrm{H}, \mathrm{CH}), 7.20-7.32(5 \mathrm{H}, \mathrm{m}, \mathrm{Ar}), 7.43-7.51(2 \mathrm{H}, \mathrm{m}$, $\mathrm{Ar})$, 7.94-8.01 (2H, m, Ar) ppm; minor diastereoisomer: $\delta 2.36\left(\mathrm{~s}, 3 \mathrm{H}, \mathrm{CH}_{3}\right), 2.38\left(\mathrm{~s}, 3 \mathrm{H}, \mathrm{CH}_{3}\right), 3.99(\mathrm{~s}, 1 \mathrm{H}, \mathrm{CH})$, 7.20-7.32 (5H, m, Ar), 7.38-7.43 (2H, m, Ar), 7.87-7.94 (2H, m, Ar) ppm.

2-(3-Bromophenyl)-4-methyl-7-oxo-6-phenyl-5,6-diazaspiro[2.4]hept-4-ene-1,1-dicarbonitrile (3p). White solid; $2.39 \mathrm{~g}$ (59\%); diastereomeric ratio 3:1; mp 194-196 ${ }^{\circ} \mathrm{C}$ (lit. ${ }^{43} \mathrm{mp} 198-200{ }^{\circ} \mathrm{C}$ ). ${ }^{1} \mathrm{H}$ NMR (DMSO-d $)_{6}$ major diastereoisomer: $\delta 1.74\left(\mathrm{c}, 3 \mathrm{H}, \mathrm{CH}_{3}\right), 4.18(\mathrm{c}, 1 \mathrm{H}, \mathrm{CH}), 7.39-7.62(\mathrm{~m}, 5 \mathrm{H}, \mathrm{Ar}), 7.83-7.93(\mathrm{~m}, 3 \mathrm{H}, \mathrm{Ar}), 8.03(\mathrm{c}, 1 \mathrm{H}$, Ar) ppm; minor diastereoisomer: $\delta 2.33\left(\mathrm{c}, 3 \mathrm{H}, \mathrm{CH}_{3}\right), 4.94(\mathrm{c}, 1 \mathrm{H}, \mathrm{CH}), 7.20-7.42(\mathrm{~m}, 6 \mathrm{H}, \mathrm{Ar}), 7.68(\mathrm{~d}, J 7.5 \mathrm{~Hz}$, $2 \mathrm{H}, \mathrm{Ar}), 7.96$ (c, 1H, Ar) ppm.

4-Methyl-2-(4-nitrophenyl)-7-oxo-6-phenyl-5,6-diazaspiro[2.4]hept-4-ene-1,1-dicarbonitrile (3q). White solid; $1.30 \mathrm{~g}$ (38\%); diastereomeric ratio 3:2; $\mathrm{mp} 169-172{ }^{\circ} \mathrm{C}$ (lit. ${ }^{43} \mathrm{mp} 161-163{ }^{\circ} \mathrm{C}$ ). ${ }^{1} \mathrm{H}$ NMR (DMSO-d 6 ) major diastereoisomer: $\delta 1.70\left(\mathrm{~s}, 3 \mathrm{H}, \mathrm{CH}_{3}\right), 4.33(\mathrm{~s}, 1 \mathrm{H}, \mathrm{CH}), 7.26-7.32(\mathrm{~m}, 1 \mathrm{H}, \mathrm{Ar}), 7.49-7.56(\mathrm{~m}, 2 \mathrm{H}, \mathrm{Ar}), 7.80(\mathrm{~d}, J 8.7$ $\mathrm{Hz}, 2 \mathrm{H}, \mathrm{Ar}), 7.88-7.94(\mathrm{~m}, 2 \mathrm{H}, \mathrm{Ar}), 8.33(\mathrm{~d}, J 8.7 \mathrm{~Hz}, 2 \mathrm{H}, \mathrm{Ar}) \mathrm{ppm}$; minor diastereoisomer: $\delta 2.27\left(\mathrm{~s}, 3 \mathrm{H}, \mathrm{CH}_{3}\right)$, $5.10(\mathrm{~s}, 1 \mathrm{H}, \mathrm{CH}), 7.21-7.26(\mathrm{~m}, 1 \mathrm{H}, \mathrm{Ar}), 7.42-7.49(\mathrm{~m}, 2 \mathrm{H}, \mathrm{Ar}), 7.76-7.85(\mathrm{~m}, 2 \mathrm{H}, \mathrm{Ar}), 7.80(\mathrm{~d}, J 8.7 \mathrm{~Hz}, 2 \mathrm{H}, \mathrm{Ar})$, $8.33(\mathrm{~d}, J 8.7 \mathrm{~Hz}, 2 \mathrm{H}, \mathrm{Ar}) \mathrm{ppm}$.

4-Methyl-2-(3-methoxyphenyl)-7-oxo-6-phenyl-5,6-diazaspiro[2.4]hept-4-ene-1,1-dicarbonitrile (3r). White solid; $2.28 \mathrm{~g}$ (64\%); diastereomeric ratio 6:1; $\mathrm{mp} 188-190{ }^{\circ} \mathrm{C} .{ }^{1} \mathrm{H}$ NMR (DMSO- $d_{6}$ ) major diastereoisomer: $\delta 1.78$ $\left(\mathrm{s}, 3 \mathrm{H}, \mathrm{CH}_{3}\right), 3.78\left(\mathrm{~s}, 3 \mathrm{H}, \mathrm{OCH}_{3}\right), 4.02(\mathrm{~s}, 1 \mathrm{H}, \mathrm{CH}), 7.20-7.32(5 \mathrm{H}, \mathrm{m}, \mathrm{Ar}), 7.34-7.52(2 \mathrm{H}, \mathrm{m}, \mathrm{Ar}), 7.88-7.96(2 \mathrm{H}, \mathrm{m}$, Ar) ppm; ${ }^{13} \mathrm{C}$ NMR (DMSO-d $)_{6}$ : $\delta$ 16.6, 20.4, 40.7, 44.9, 55.5, 111.1, 111.7, 114.8, $118.5(2 \mathrm{C}), 119.8,123.7,129.3$ (2C), 129.6, 130.9, 132.6, 133.4, 150.8, 159.8, 165.1. IR (KBr): 768, 1248, 1424, 1480, 1608, 1708, $2254 \mathrm{~cm}^{-1}$. HRMS (ESI) $357.1360[\mathrm{M}+\mathrm{H}]^{+}$, calcd for $\mathrm{C}_{21} \mathrm{H}_{17} \mathrm{~N}_{4} \mathrm{O}_{2}: 357.1352$. 


\section{Acknowledgements}

The reported study was funded by RFBR according to the research project № 18-33-00301.

\section{References}

1. Yan, M.; Kawamata, Y.; Baran, P. S. Chem. Rev. 2017, 117, 13230. https://doi.org/10.1021/acs.chemrev.7b00397

2. Jiang, Y.; Xu, K.; Zeng, C. Chem. Rev. 2018, 118, 4485. https://doi.org/10.1021/acs.chemrev.7b00271

3. Vereshchagin, A. N. Russ. Chem. Bull. 2017, 66, 1765. https://doi.org/10.1007\%2Fs11172-017-1950-1

4. Shatskiy, A.; Lundberg, H.; Karkas, M. D. ChemElectroChem 2019, 6, doi: 10.1002/celc.201900435 https://onlinelibrary.wiley.com/doi/full/10.1002/celc.201900435

5. Liang, S.; Xu, K.; Zeng, C. C.; Tian, H. Y.; Sun, B. G. Adv. Synth. Catal. 2018, 360, 4266. https://onlinelibrary.wiley.com/doi/full/10.1002/adsc.201800519

6. Ogibin, Y. N.; Elinson, M. N.; Nikishin, G. I. Russ. Chem. Rev. 2009, 78, 89. http://dx.doi.org/10.1070/RC2009v078n02ABEH003886

7. Francke, R.; Little, R. D. Chem. Soc. Rev. 2014, 43, 2492. https://pubs.rsc.org/en/content/articlelanding/2014/CS/c3cs60464k\#!divAbstract

8. Shono, T. Tetrahedron 1984, 40, 811. https://doi.org/10.1016/S0040-4020(01)91472-3

9. Steckhan, E. Top. Curr. Chem. 1987, 142, 1. https://link.springer.com/chapter/10.1007/3-540-17871-6 11

10. Elinson, M. N.; Dorofeeva, E. O.; Vereshchagin, A. N.; Nikishin, G. I. Russ. Chem. Rev. 2015, 84, 485. http://dx.doi.org/10.1070/RCR4465

11. Elinson, M. N.; Feducovich, S. K.; Zaimovskaya, T. A.; Vereshchagin, A. N.; Nikishin, G. I. Russ. Chem. Bull., Int. Ed. 2003, 52, 2241.

https://link.springer.com/article/10.1023\%2FB\%3ARUCB.0000011885.10104.da

12. Elinson, M. N.; Feducovich, S. K.; Zaimovskaya, T. A.; Vereshchagin, A. N.; Gorbunov, S. V.; Nikishin, G. I. Russ. Chem. Bull., Int. Ed. 2005, 54, 1593. https://link.springer.com/article/10.1007\%2Fs11172-006-0008-6

13. Elinson, M. N.; Feducovich, S. K.; Starikova, Z. A.; Vereshchagin, A. N.; Nikishin, G. I. Tetrahedron 2004, 60, 11743.

https://doi.org/10.1016/i.tet.2004.10.003

14. Elinson, M. N.; Feducovich, S. K.; Zaimovskaya, T. A.; Vereshchagin, A. N.; Nikishin, G. I. Russ. Chem. Bull., Int. Ed. 2005, 54, 673.

https://doi.org/10.1007/s11172-005-0304-6

15. Vereshchagin, A. N.; Elinson, M. N.; Egorov, M. P. RSC Adv. 2015, 5, 98522. https://doi.org/10.1039/C5RA19690F

16. Bityukov, O. V.; Matveeva, O. K.; Vil', V. A.; Kokorekin, V. A.; Nikishin, G. I.; Terent'ev, A. O. J. Org. Chem., 2019, 84, 1448. https://doi.org/10.1021/acs.joc.8b02791

17. Wang, L.; Gao, J.; Wan, L.; Wang, Y.; Yao, C. Res. Chem. Intermed. 2015, 41, 2775. 
https://doi.org/10.1007/s11164-013-1387-6

18. Elinson, M. N.; Vereshchagin, A. N.; Ryzhkov, F. V. Chem. Rec. 2016, 16, 1950. https://doi.org/10.1002/tcr.201600044

19. Yao, C.; Wang, Y.; Li, T.; Yu, C.; Li, L.; Wang, C. Tetrahedron 2013, 69, 10593. http://dx.doi.org/10.1016/i.tet.2013.10.056

20. Elinson, M. N.; Vereshchagin, A. N.; Ryzhkov, F. V. Curr. Org. Chem. 2017, 21, 1427. http://dx.doi.org/10.2174/1385272820666161017170200

21. Brunton, L. L.; Chabner, B. A.; Knollmann, B. C. Keith, L. P. Goodman \& Gilman's the Pharmacological Basis of Therapeutics, 12th ed.; The McGraweHill Companies: New York, NY, 2011.

22. Johns, M. W. Drugs 1975, 9, 448. https://doi.org/10.2165/00003495-197509060-00004

23. Veisi, H.; Maleki, A.; Omrani, Z.; Lotfi, S. RSC Adv. 2014, 4, 55313. https://doi.org/10.1039/c4ra08251f

24. Dundee, J. W.; Mcilroy, P. D. A. Anaesthesia 1982, 37, 726. https://doi.org/10.1111/i.1365-2044.1982.tb01310.x

25. Galati, E. M.; Monforte, M. T.; Miceli, N.; Raneri, E. Farmaco 2001, 56, 459. https://doi.org/10.1016/S0014-827X(01)01062-X

26. Lomlin, L.; Einsiedel, J.; Heinemann, F.W.; Meyer, K.; Gmeiner, P. J. Org. Chem. 2008, 73, 3608. https://doi.org/10.1021/jo702573z

27. Vereshchagin, A. N., Elinson, M. N.; Tretyakova, E. O.; Zaimovskaya, T. A.; Stepanov, N. O.; Gorbunov, S. V.; Belyakov, P. A.; Nikishin, G. I. Tetrahedron 2012, 68, 1198. https://doi.org/10.1016/j.tet.2011.11.057

28. Vereshchagin, A. N.; Elinson, M. N.; Dorofeeva, E. O.; Bushmarinov, I. S.; Gorbunov, S. V.; Belyakov, P. A.; Chizhov, A. O.; Nikishin, G. I. J. Mol. Cat. A: Chem. 2012, 363-364, 69. https://doi.org/10.1016/j.molcata.2012.05.018

29. Dorofeeva, E. O.; Elinson, M. N.; Vereshchagin, A. N.; Stepanov, N. O.; Bushmarinov, I. S.; Belyakov, P. A.; Sokolova, O. O.; Nikishin, G. I. RSC Adv. 2012, 2, 4444.

https://doi.org/10.1039/C2RA20078C

30. Sandanayaka, V.P.; Prashad, A.S.; Yang, Y.; Williamson, R.T.; Lin, Y.I.; Mansour, T.S. J. Med. Chem. 2003, 46, 2569.

https://doi.org/10.1021/jm034056q

31. Laroche, C.; Behr, J.-B.; Szymoniak, J.; Bertus, P.; Schutz, C.; Vogel, P.; Plantier-Royon, R. Bioorg. Med. Chem. 2006, 14, 4047. https://doi.org/10.1016/j.bmc.2006.02.005

32. Varvounis, G. Adv. Het. Chem. 2009, 98, 143. https://doi.org/10.1016/S0065-2725(09)09802-X

33. Yoshida, H.; Yanai, H.; Namiki, Y.; Fukatsu-Sasaki, K.; Furutani, N.; Tada, N. CNS Drug Rev., 2006, $12,9$. https://doi.org/10.1111/i.1527-3458.2006.00009.x

34. Li, W. C.; Zhang, Z. Z.; Zeng, C. C.; Hu, L. M.; Tian, H. Y. Curr. Org. Synth. 2014, 11, 454. https://doi.org/10.2174/15701794113109990002

35. Elinson, M. N.; Dorofeeva, E. O.; Vereshchagin, A. N.; Nasybullin, R. F.; Egorov, M. P. Catal. Sci. Technol., 2015, 5, 2384. https://doi.org/10.1039/c4cy01681e

36. Itokawa, M.; Miyata, T.;. Arai, M. Eur. Pat. Appl., EP 2189537 A1, 2010. 
https://depatisnet.dpma.de/DepatisNet/depatisnet?action=pdf\&docid=EP000002189537A1

37. Elinson, M. N.; Feducovich, S. K.; Vereshchagin, A. N.; Dorofeev, A. S.; Dmitriev, D. E.; Nikishin, G. I. Russ.

Chem. Bull., Int. Ed. 2003, 52, 2235.

https://link.springer.com/article/10.1023\%2FB\%3ARUCB.0000011884.08799.a7

38. Gao, W. J.; Li, W. C.; Zeng, C. C.; Tian, H. Y.; Hu, L. M.; Little, R. D. J. Org. Chem., 2014, 79, 9613.

https://doi.org/10.1021/jo501736w

39. Jiang, Y.; Wang, Q. Q.; Liang, S.; Hu, L. M.; Little, R. D. J. Org. Chem. 2016, 81, 4713. https://10.1021/acs.joc.6b00615

40. Elinson, M. N.; Dorofeeva, E. O.; Vereshchagin, A. N.; Korshunov, A. D.; Egorov, M. P. Res. Chem. Intermed. 2016, 42, 2191.

https://doi.org/10.1007/s11164-015-2142-y

41. Abaszadeh, M.; Seifi, M. Org. Biomol. Chem. 2014, 12, 7859.

https://doi.org/10.1039/c4ob01305k

42. Elinson, M. N.; Vereshchagin, A. N.; Stepanov, N. O.; llovaisky, A. I.; Vorontsov, A. Y.; Nikishin, G. I.

Tetrahedron 2009, 65, 6057.

https://doi.org/10.1016/j.tet.2009.05.062

43. Vereshchagin, A. N.; Elinson, M. N.; Korshunov, A. D.; Korolev, V. A.; Egorov, M. P. Heterocycl. Commun. 2015, 21, 355.

https://doi.org/10.1515/hc-2015-0141 